\title{
Joint Design of GNSS Signal and Message Structure for Gallleo 2nd Ceneration
}

Lorenzo Ortega Espluga, TesA Charly Poulliat, Marie-Laure Boucheret, Toulouse INP

Marion Aubault, CNES

Hanaa Al Bitar, Thales Alenila Space

ThalesAlenía $\ldots$. cnes ... TêSA 
- Context: Galileo 2nd Generation (G2G)

- Goal:

- Improve the acquisition phase

- Improve the receiver sensitivity

- Reduce the TTFF*

- Opportunity:

- Design a new signal to improve the acquisition phase

- Wayforward:

- Design of a new modulation

- Design of a new family of $\mathrm{PRN}^{*}$ code

- Co-design of the message structure and the channel coding scheme

$\mathrm{PRN}^{*}=$ Pseudo Random Noise

TTFF $^{*}=$ Time To First Fix 
- Introduction \& background

- Design a new modulation for fast acquisition:

- BCS

- Design new PRN codes for fast acquisition:

- Random codes

- Co-design of the message structure and channel coding:

- Maximum distance separable codes(MDS)

- Full diversity Codes

- Conclusion

- Future Lines 
- Introduction \& background

- Design a new modulation for fast acquisition:

- BCS

- Design new PRN codes for fast acquisition:

- Random codes

- Co-design of the message structure and channel coding :

- Maximum distance separable codes(MDS)

- Full diversity Codes

- Conclusion

- Future Lines 
New signal to improve the TTFF and the receiver sensitivity

- What does improving the TTFF involve?

- What does improving the receiver sensitivity involve? 
New signal to improve the TTFF and the receiver sensitivity

- What does improving the TTFF involve?

$$
T T F F=T_{\text {warm-up }}+T_{\text {acq }}+T_{\text {track }}+T_{C E D}+T_{P V T}
$$

- What does improving the receiver sensitivity involve?

- Acquisition

- Tracking

- Data Demodulation ... 
New signal to improve the TTFF and the receiver sensitivity

\section{Spreading} Modulation

\section{PRN Code}

Channel

Coding
Message Structure 
New signal to improve the TTFF and the receiver sensitivity

\section{Spreading} Modulation

\section{PRN Code}

Channel

Coding

Message Structure

\section{TTFF}

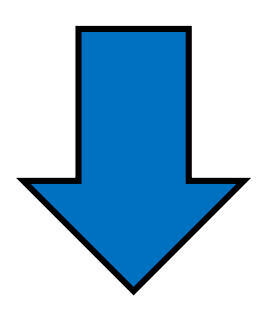


- Introduction \& background

- Design a new modulation for fast acquisition:

$\circ$ BCS

- Design new PRN codes for fast acquisition:

- Random codes

- Co-design of the message structure and channel coding:

- Maximum distance separable codes(MDS)

- Full diversity Codes

- Conclusion

- Future Lines 


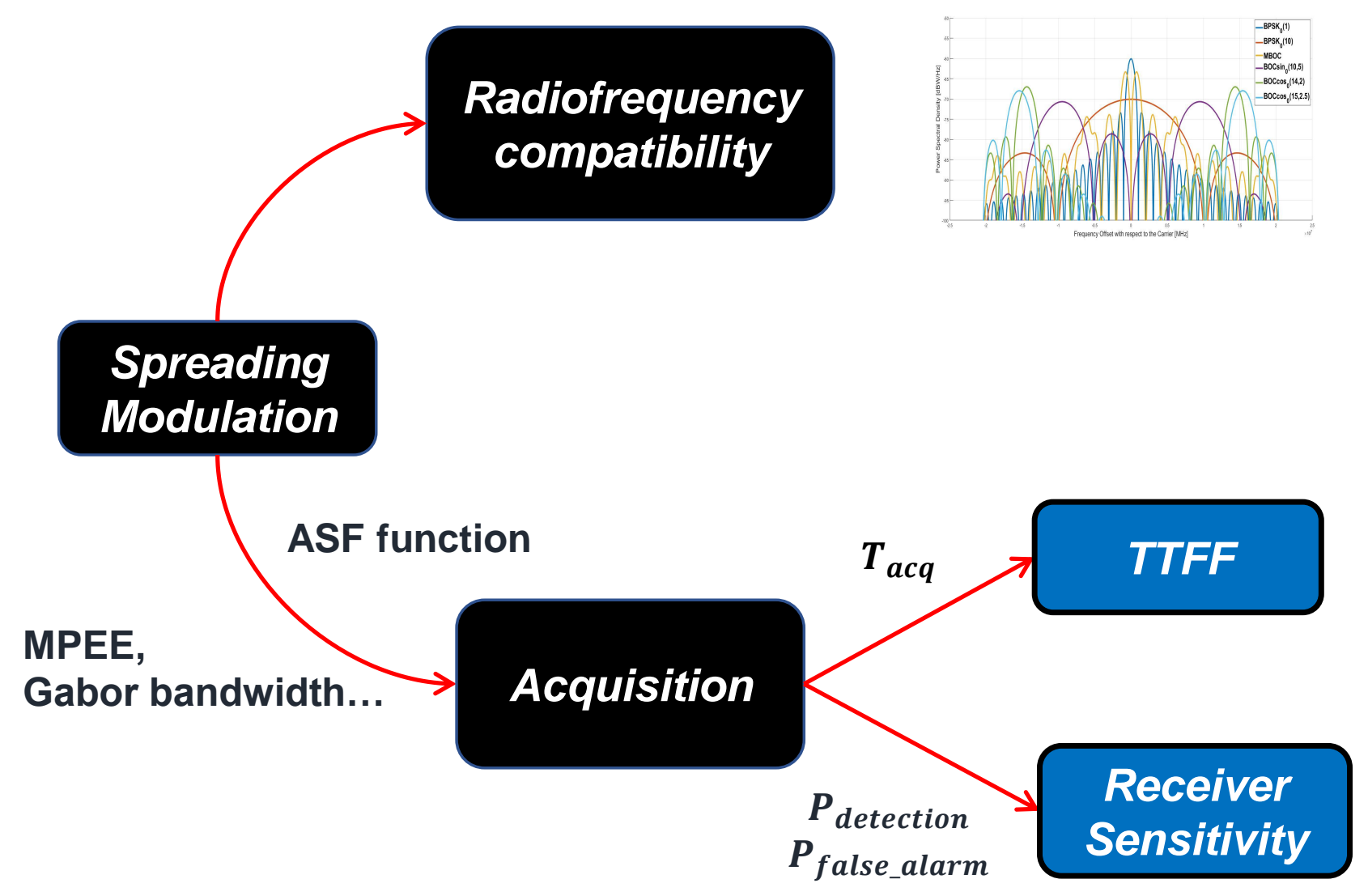




\section{- Spreading Modulation Criteria for Design}

\begin{tabular}{|c|c|}
\hline Figure of Merit & Criteria \\
\hline Radio Frequency Compatibility & SSC \\
\hline Correlation Properties & ACF \\
\hline Resistance Against Multipath & MPEE \\
\hline Ranging Performance & Gabor bandwidth \\
\hline Anti-Jamming Capability & $\begin{array}{c}\text { Demodulation \& anti-jamming of narrowband } \\
\text { Code tracking \& anti-jamming of narrowband } \\
\text { Demodulation \& anti-jamming of matched } \\
\text { spectrum }\end{array}$ \\
$\begin{array}{c}\text { Code tracking \& anti-jamming of matched } \\
\text { spectrum }\end{array}$ \\
\hline
\end{tabular}




\section{Design new modulation}

- Standard spreading modulations:

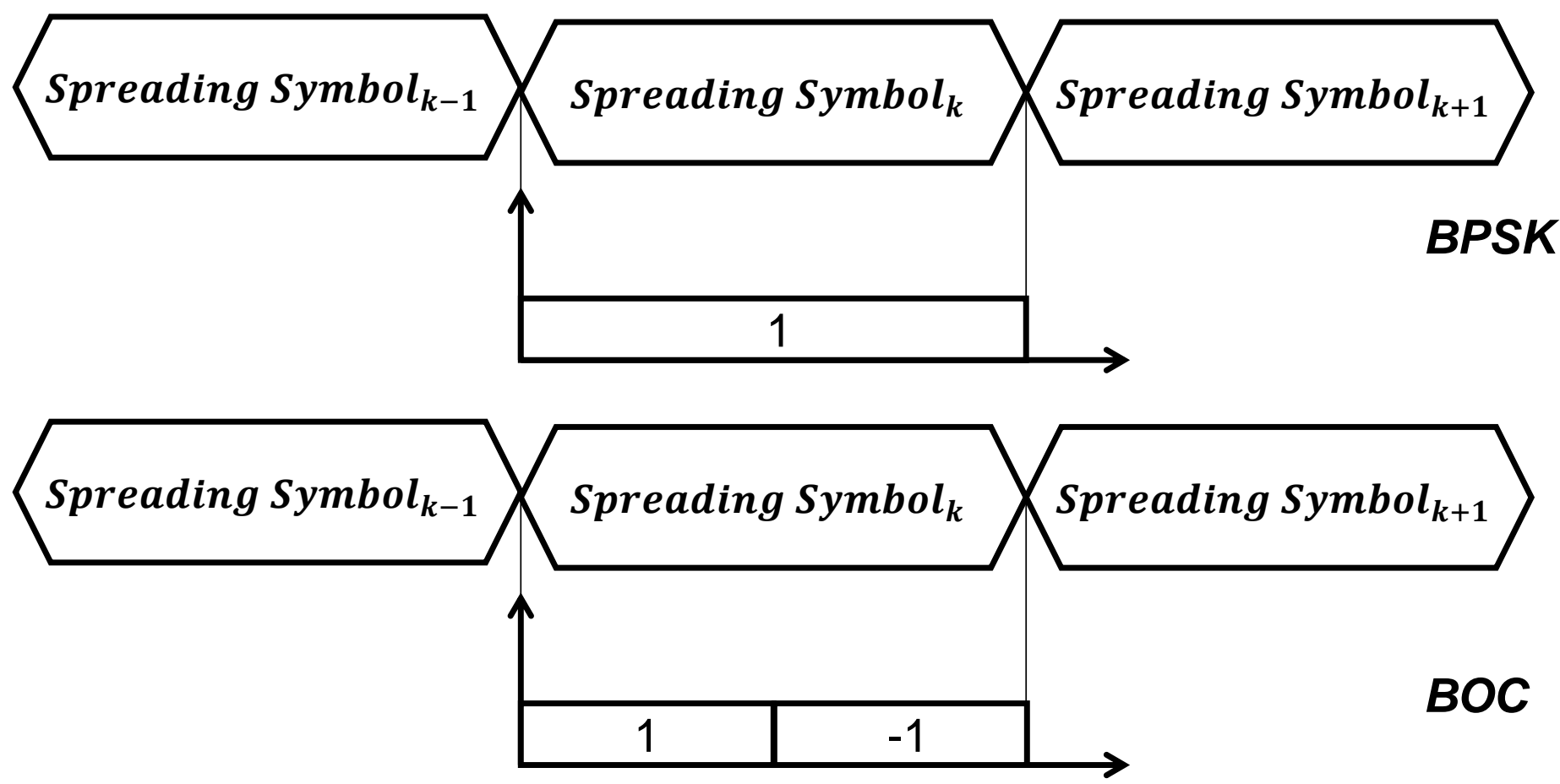


- Binary Coded Symbol (BCS)

- Spreading modulation candidate

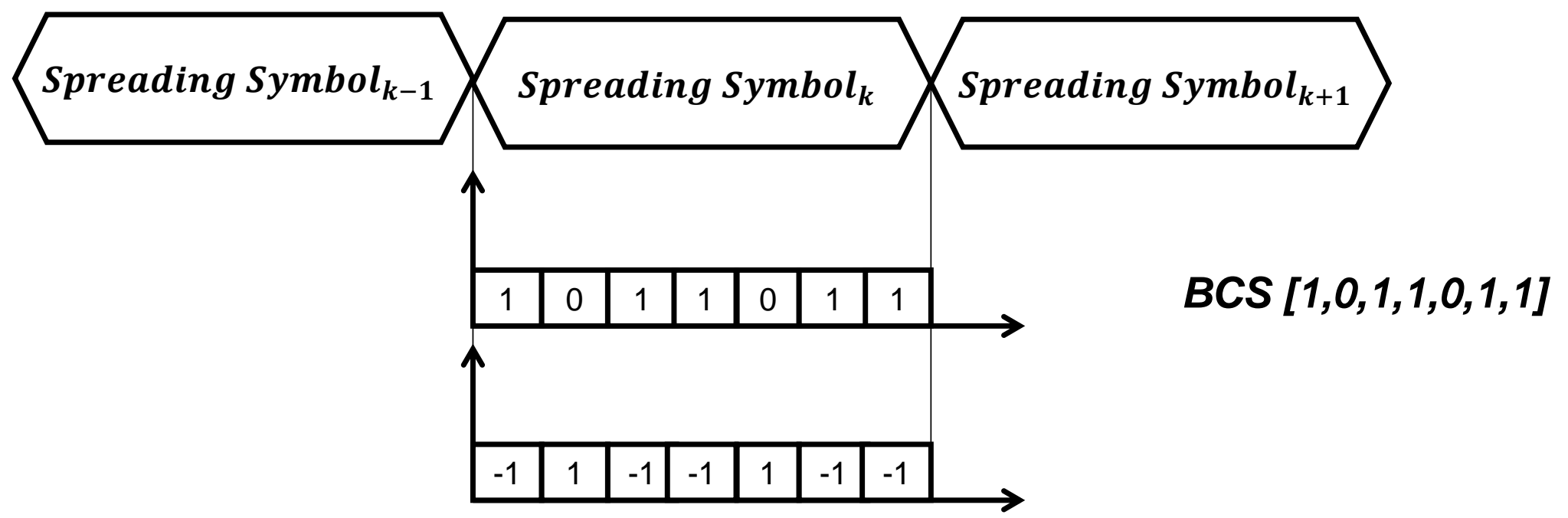


- Spreading modulation candidates

\begin{tabular}{|c|c|c|c|}
\hline \multicolumn{2}{|c|}{ Already proposed candidates } & \multicolumn{2}{|c|}{ Proposed candidates } \\
\hline $\mathrm{BOC} \cos (0.5,0.5)$ & $\operatorname{BOC\operatorname {sin}}(4,1)$, & $\mathrm{BCS}[-1,1](0.5)$ & BCS[-1,-1, -1, 1, 1](1) \\
\hline $\operatorname{BOCsin}(0.5,0.5)$ & $\operatorname{BOC} \cos (4,1)$, & BCS $[-1,1,-1](0.5)$ & BCS $[-1,1,-1](1)$ \\
\hline $\operatorname{BOC} \sin (4,0.5)$ & $\mathrm{BOC} \sin (6.5,0.5)$ & BCS $[-1,-1,-1,1,1](0.5)$ & BCS $[-1,-1,-1,1,-1](1)$ \\
\hline $\mathrm{BOC} \cos (4,0.5)$ & BOC $\cos (6.5,0.5)$ & & \\
\hline
\end{tabular}

Current L1 bandwidth spectral occupation

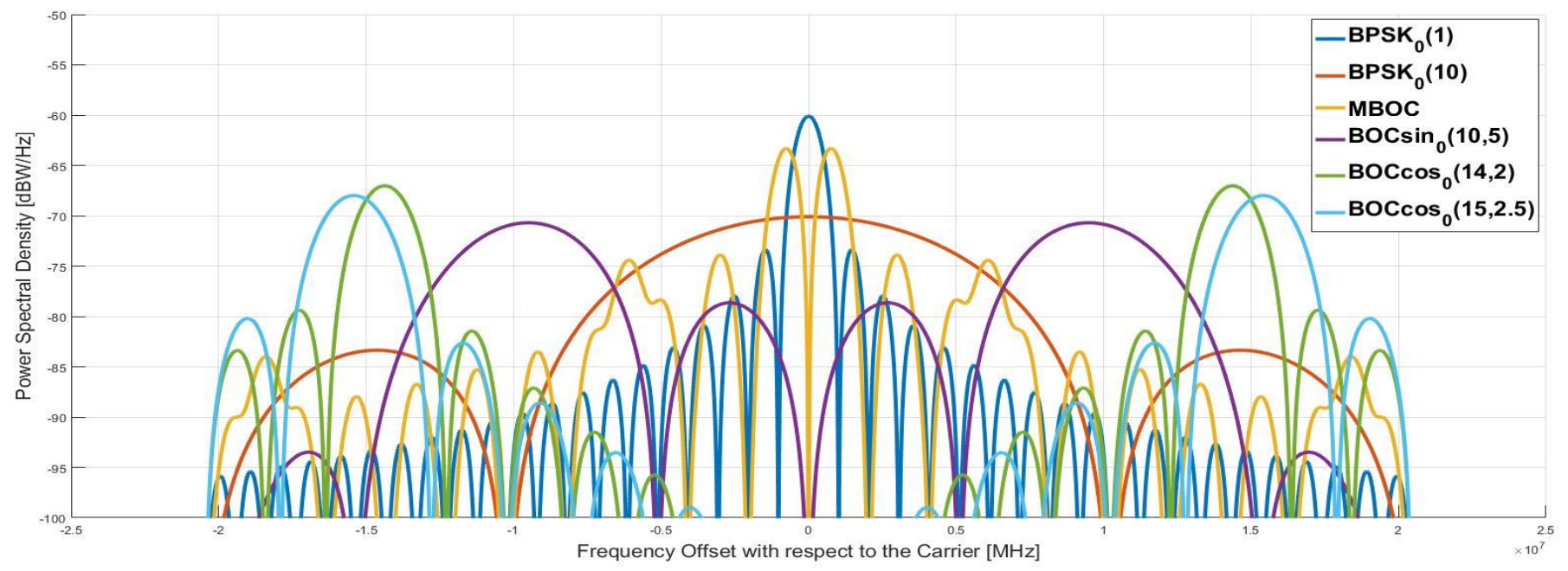


- Evaluation of the spreading modulation candidates

- Radio frequency compatibility

\begin{tabular}{|c|c|c|c|c|c|c|}
\hline & \multicolumn{6}{|c|}{ ssc Coefficients } \\
\hline$B O C \cos _{0}(6.5,0.5)$ & -88.43 & -78.27 & -79.09 & -77.49 & -90.91 & -90.29 \\
\hline BOCsin $_{0}(6.5,0.5)$ & -85.40 & -77.79 & -78.46 & -77.85 & -94.23 & -92.64 \\
\hline$B O C \cos _{0}(4,0.5)$ & -88.96 & -73.54 & -87.47 & -80.91 & -96.51 & -94.08 \\
\hline $\operatorname{BOCsin}_{0}(4,0.5)$ & -82.93 & -73.26 & -82.99 & -81.20 & -95.06 & -94.09 \\
\hline $\operatorname{BOC} \cos _{0}(4,1)$ & -85.95 & -73.66 & -84.46 & -80.46 & -93.48 & -93.83 \\
\hline Ididates $\operatorname{Bocsin}_{0}(4,1)$ & -79.92 & -73.11 & -79.98 & -81.00 & -92.04 & -93.84 \\
\hline BOC $\cos _{0}(0.5,0.5)$ & -66.12 & -70.48 & -65.27 & -83.92 & -92.96 & -93.57 \\
\hline${ }^{B O C \sin _{0}(0.5,0.5)}$ & 11 & -70.32 & -66.52 & -86.13 & -95.17 & -95.79 \\
\hline BCS[-1 1] $(0.5)$ & -63 & -70.32 & -66.52 & -86.13 & -95.17 & -95.79 \\
\hline BCS[-1 $1-1][0.5)$ & -66.75 & -70.48 & -64.86 & -83.92 & -92.94 & -93.56 \\
\hline BCS[- $\left.\begin{array}{llll}-1 & -1 & 1 & 1\end{array}\right](0.5)$ & -63.09 & -70.32 & -66.57 & -86.13 & -95.17 & -95.79 \\
\hline BCS[- $\left.\begin{array}{llll}-1 & -1 & 1 & 1\end{array}\right](1)$ & -67.39 & -70.56 & -65.50 & -83.12 & -91.68 & -92.80 \\
\hline BCS[-1 $1-1](1)$ & -71.40 & -70.89 & -68.98 & -80.90 & -88.80 & -91.90 \\
\hline BCS[- $\left.\begin{array}{llll}-1 & -1 & 1 & -1\end{array}\right](1)$ & -66.11 & -70.89 & -69.37 & -80.90 & -91.31 & -94.60 \\
\hline & $\mathrm{BPSK}_{0}(1)$ & $\mathrm{BPSK}_{0}(10)$ & MBOC & $\mathrm{BOCsin}_{0}(10,5)$ & $B O C \cos _{0}(14,2)$ & $B O C \cos _{0}(15,2.5)$ \\
\hline
\end{tabular}

More interference

Less interference 
- Evaluation of the spreading modulation candidates - Correlation properties
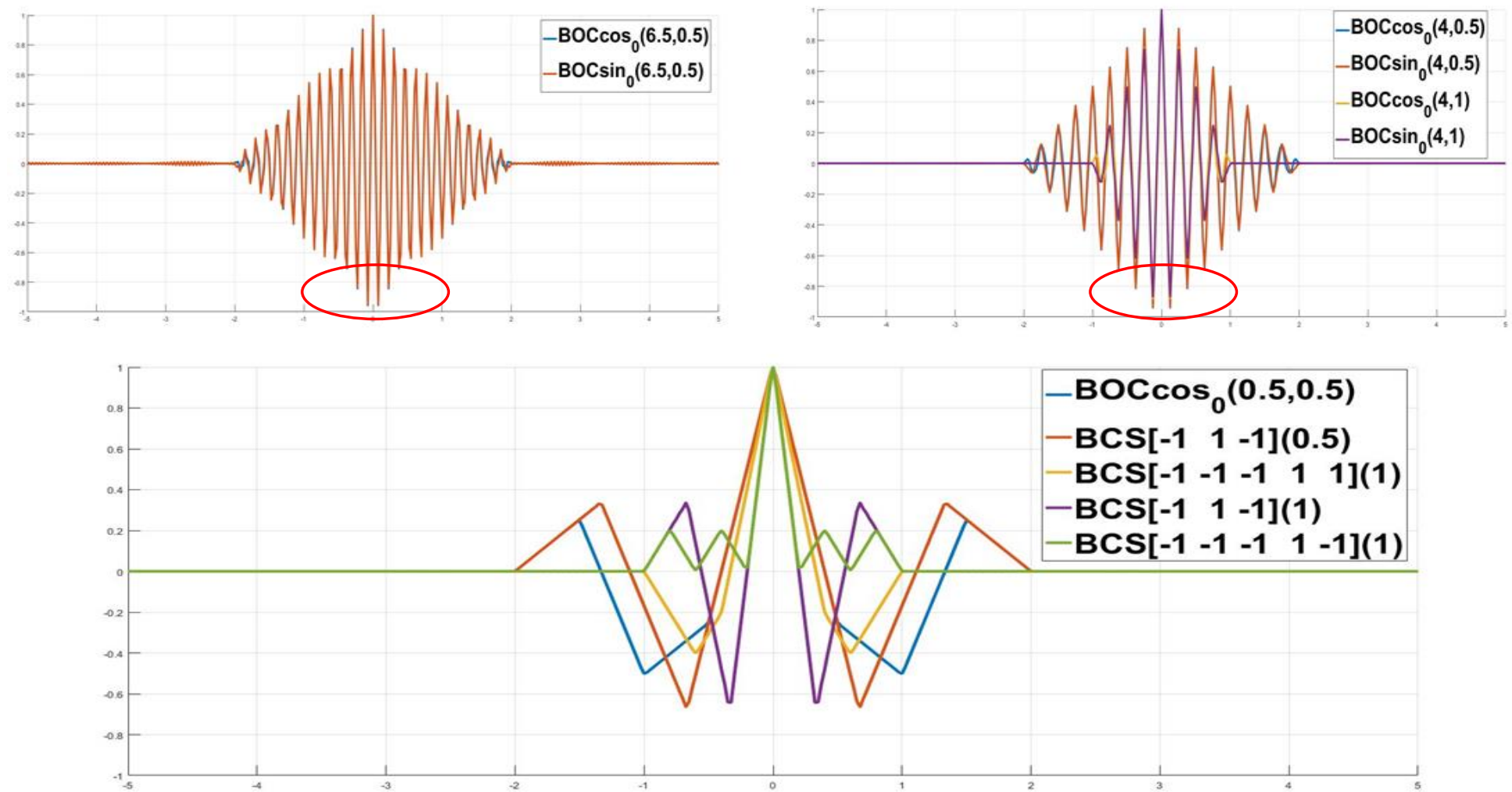
- Evaluation spreading modulation candidates - Resistance against multipath

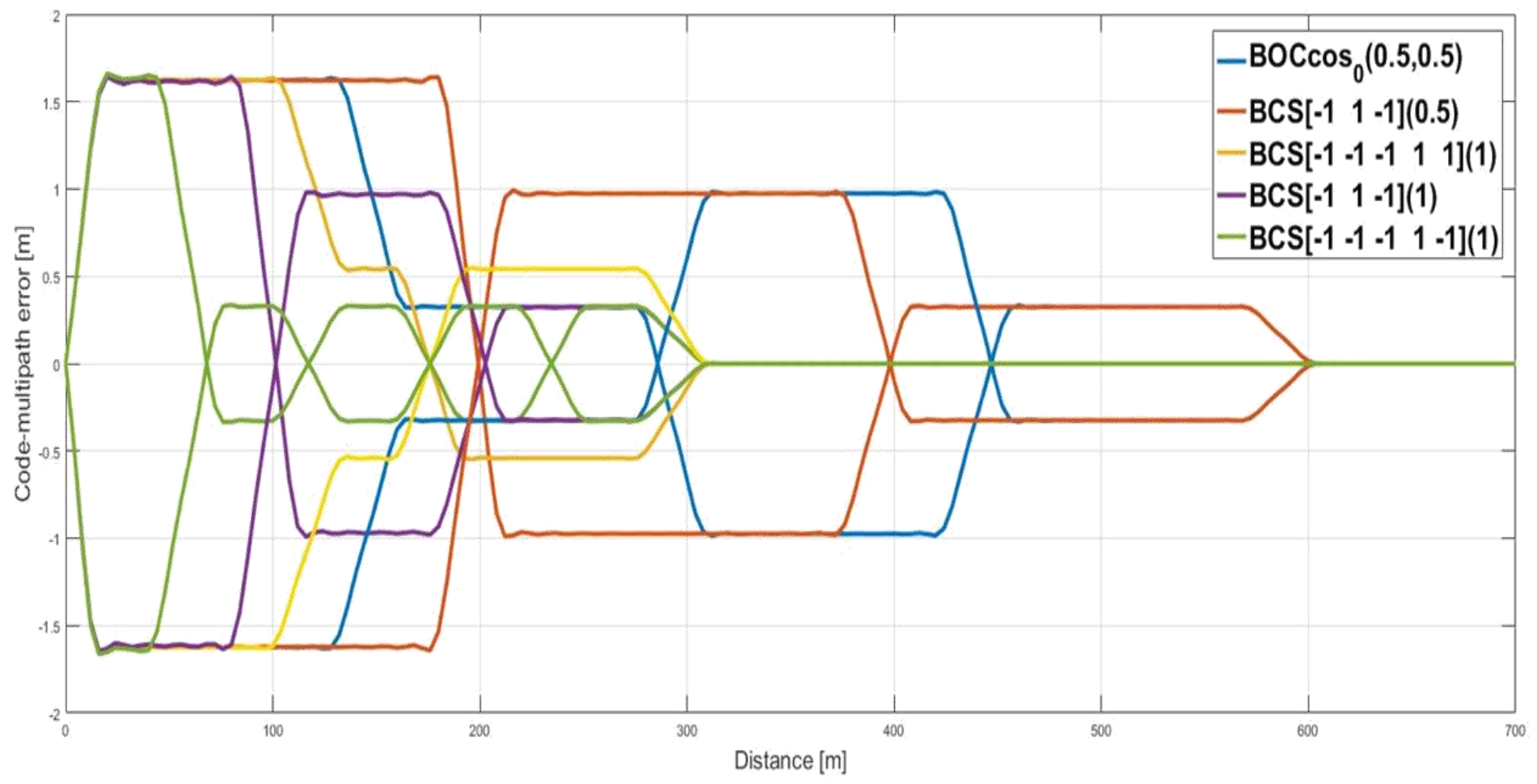


- Evaluation of the spreading modulation candidates - Ranging Performance

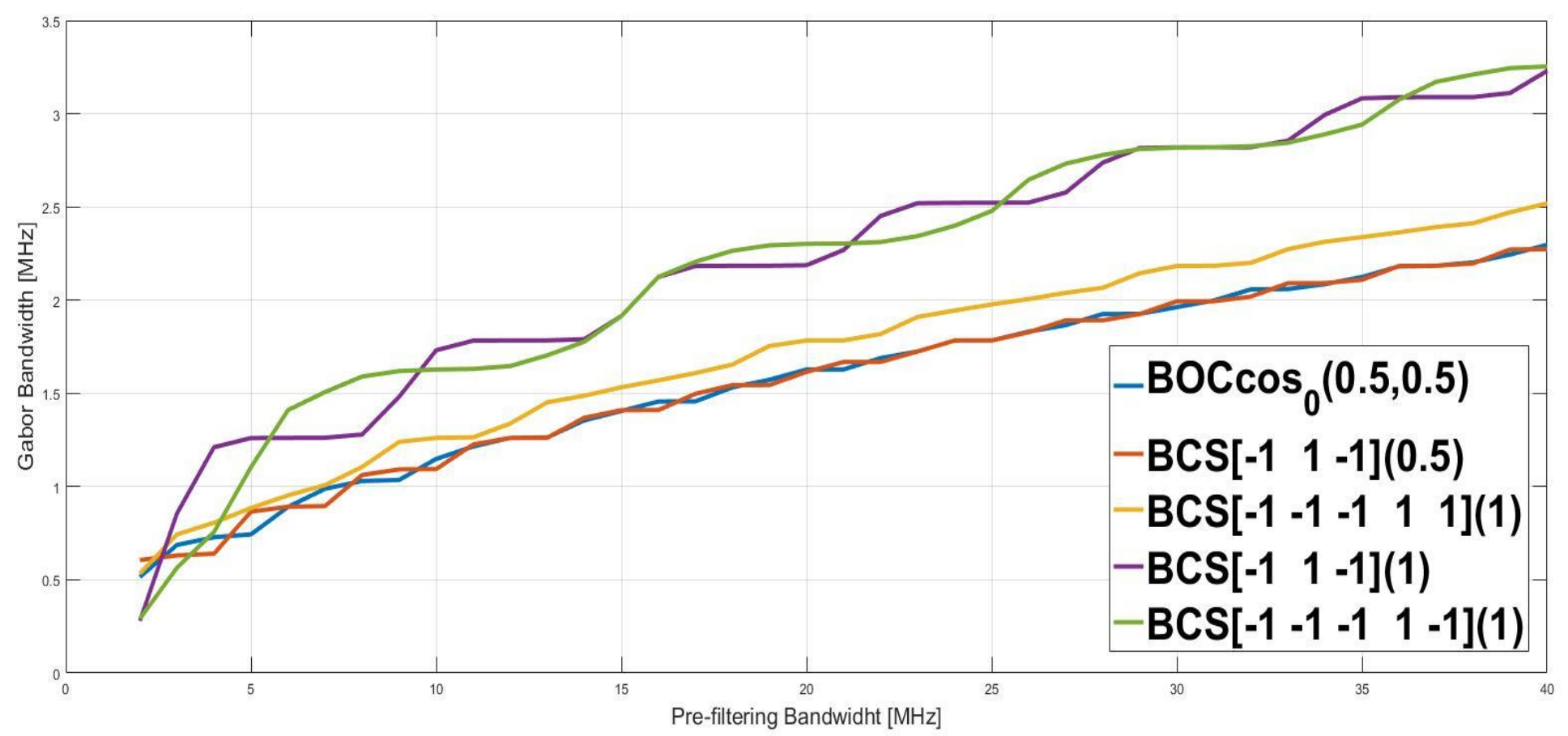


- Evaluation of the spreading modulation candidates - Anti-Jamming coefficients

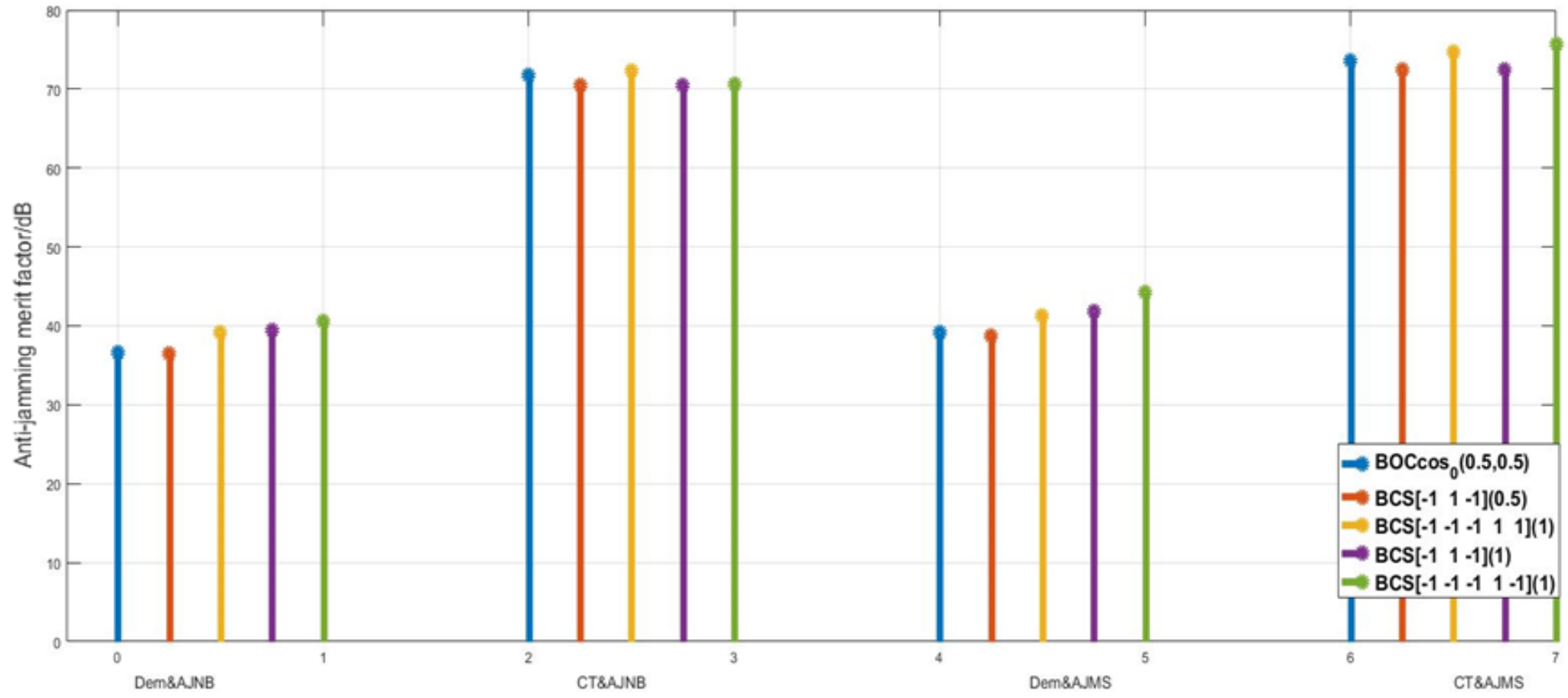


- Evaluation of the spreading modulation candidates - Polygon performance

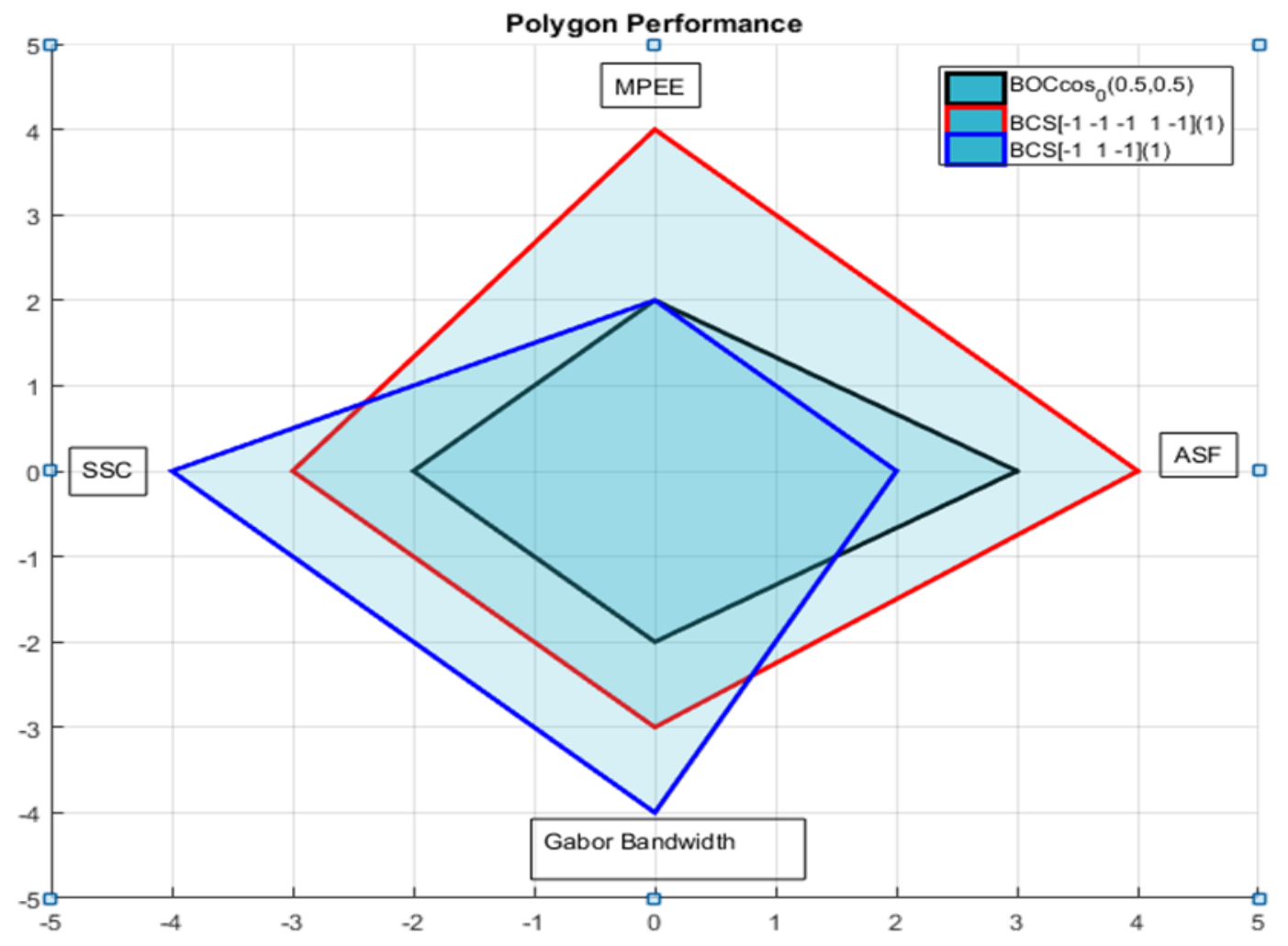


- Evaluation of the spreading modulation candidates

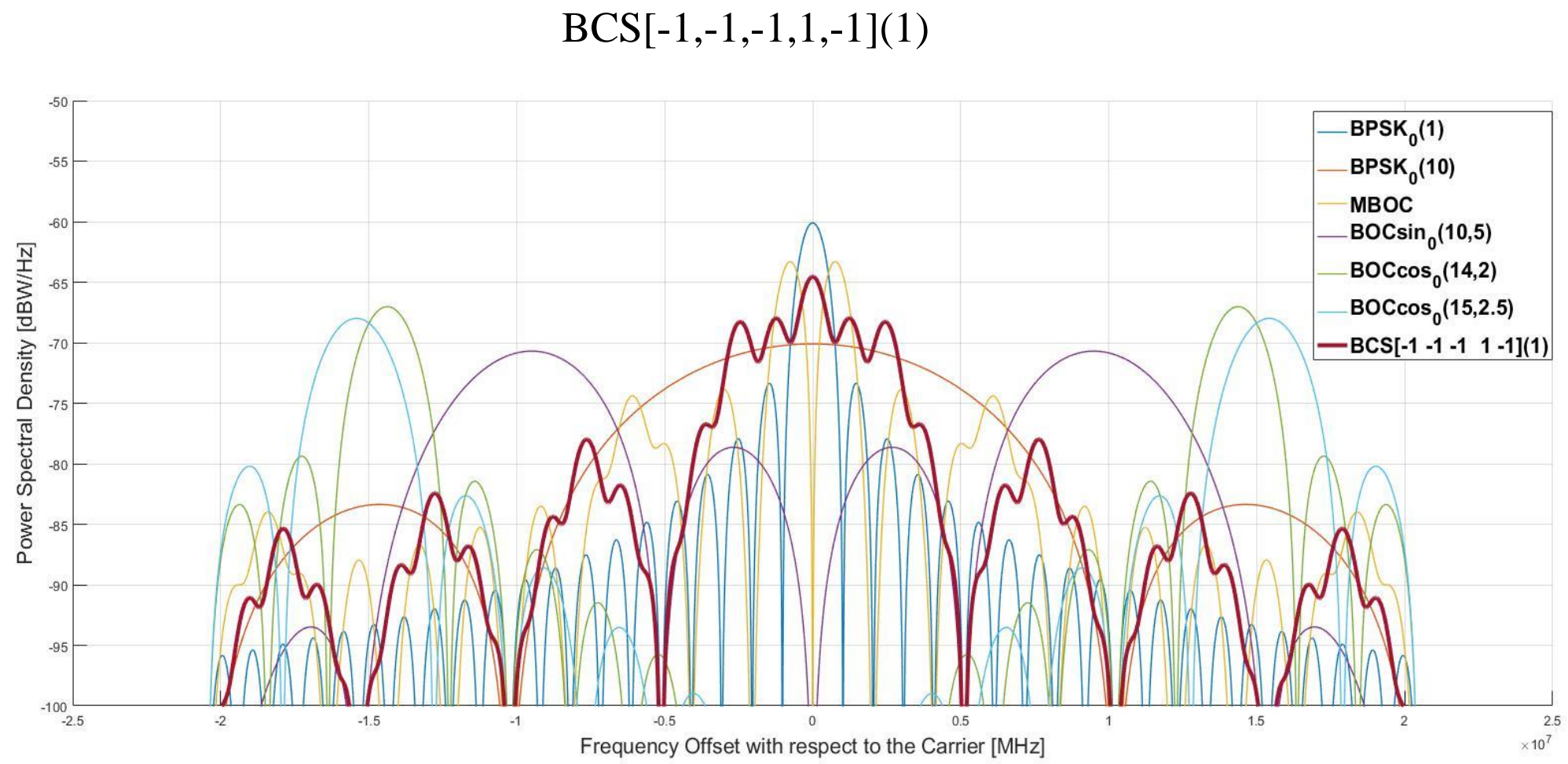


- Introduction \& background

- Design a new modulation for fast acquisition:

- BCS

- Design new PRN codes for fast acquisition:

- Random codes

- Co-design of the message structure and channel coding :

- Maximum distance separable codes(MDS)

- Full diversity Codes

- Conclusion

- Future Lines 


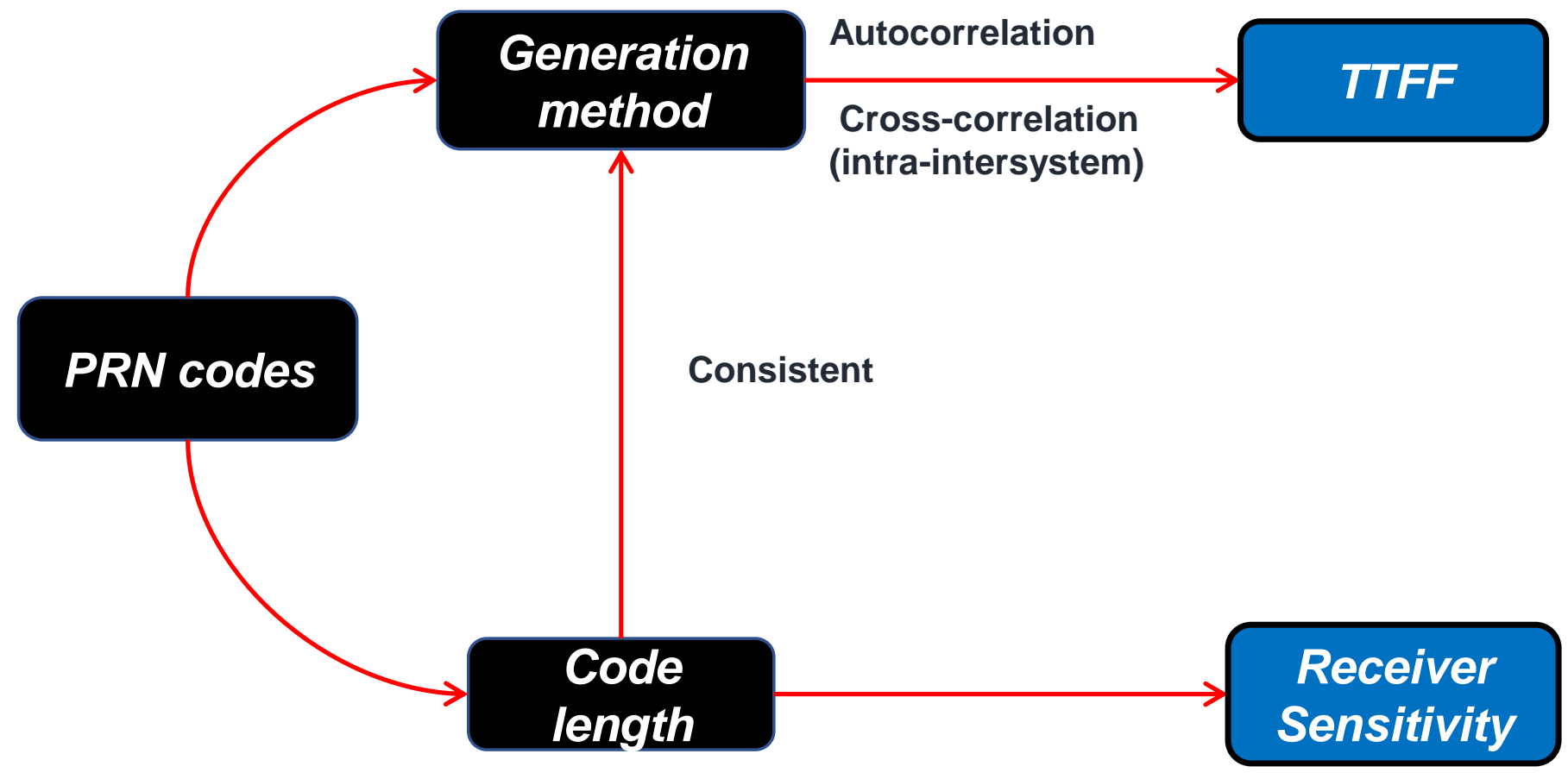




\section{Design new PRN codes}

\section{- PRN codes criteria for design}

\begin{tabular}{|c|c|}
\hline Criteria & Equations \\
\hline Acquisition Criterion & $\begin{array}{l}M E W S D^{M P}=\operatorname{mean}\left(\sum_{n_{\text {foffs }}} \sum_{A C F^{e}\left(l, f_{\text {offss }}\right)>\Phi_{\min }}^{N-1}\left(A C F^{e}\left(l, f_{\text {offs }}\right)-\Phi_{\min }\right)^{2}\right) \\
M E W S D^{C T}{ }_{i, j}=\operatorname{mean}\left(\sum_{n_{f o f f s}} \sum_{\substack{l=1 \\
C C^{e}\left(l, f_{\text {offs }}\right)>\phi_{\min }}}^{N-1}\left(C C^{e}\left(l, f_{\text {offs }}\right)-\Phi_{\min }\right)^{2}\right)\end{array}$ \\
\hline Tracking Criterion & $\begin{array}{c}M F_{i}^{M P}=\frac{1}{n_{\text {foffs }}}\left(\sum_{n_{\text {foffs }}(}\left(\sum_{l=1,2, N-2, N-1}\left(A C_{i}^{e}\left(l, f_{\text {offs }}\right)\right)^{2}\right)\right. \\
M F_{i, j}{ }^{C T}=\frac{1}{n_{\text {foffs }}}\left(\sum_{n_{\text {foffs }}}\left(\sum_{l=0}^{N-1}\left(C C_{i, j}{ }^{e}\left(l, f_{\text {offs }}\right)\right)^{2}\right)\right.\end{array}$ \\
\hline $\begin{array}{l}\text { Robustness Against Narrow-Band } \\
\text { Interferences Criterion }\end{array}$ & $E L W=10 \log \left(\frac{1}{n} \sum_{\substack{k=-\frac{n}{2} \\
A_{k}>\sqrt{n}}}^{\frac{2}{2}}\left(A_{k}-\sqrt{n}\right)^{2}\right)$ \\
\hline
\end{tabular}


- PRN codes assessment

Criteria

\begin{tabular}{|c|}
\hline$A M E W S D^{M P}$ \\
\hline$A M E W S D^{C T}$ \\
\hline$A M F^{M P}$ \\
\hline$A M F^{C T}$ \\
\hline$A E L W$ \\
\hline
\end{tabular}

Candidate

Gold Codes Kasami Codes Random Sequences Random Sequence Init Gold Codes Random Sequence Init Kasami Codes

$$
R_{i}=\sum_{j=1}^{5}-w_{j} \frac{\overline{c v_{j}}+c v_{i, j}}{\overline{c v_{j}}} \text { for } i=1,2, \ldots K
$$

$$
\text { Weight }=[0.2,0.2,0.2,0.2,0.2]
$$


- PRN codes assessment

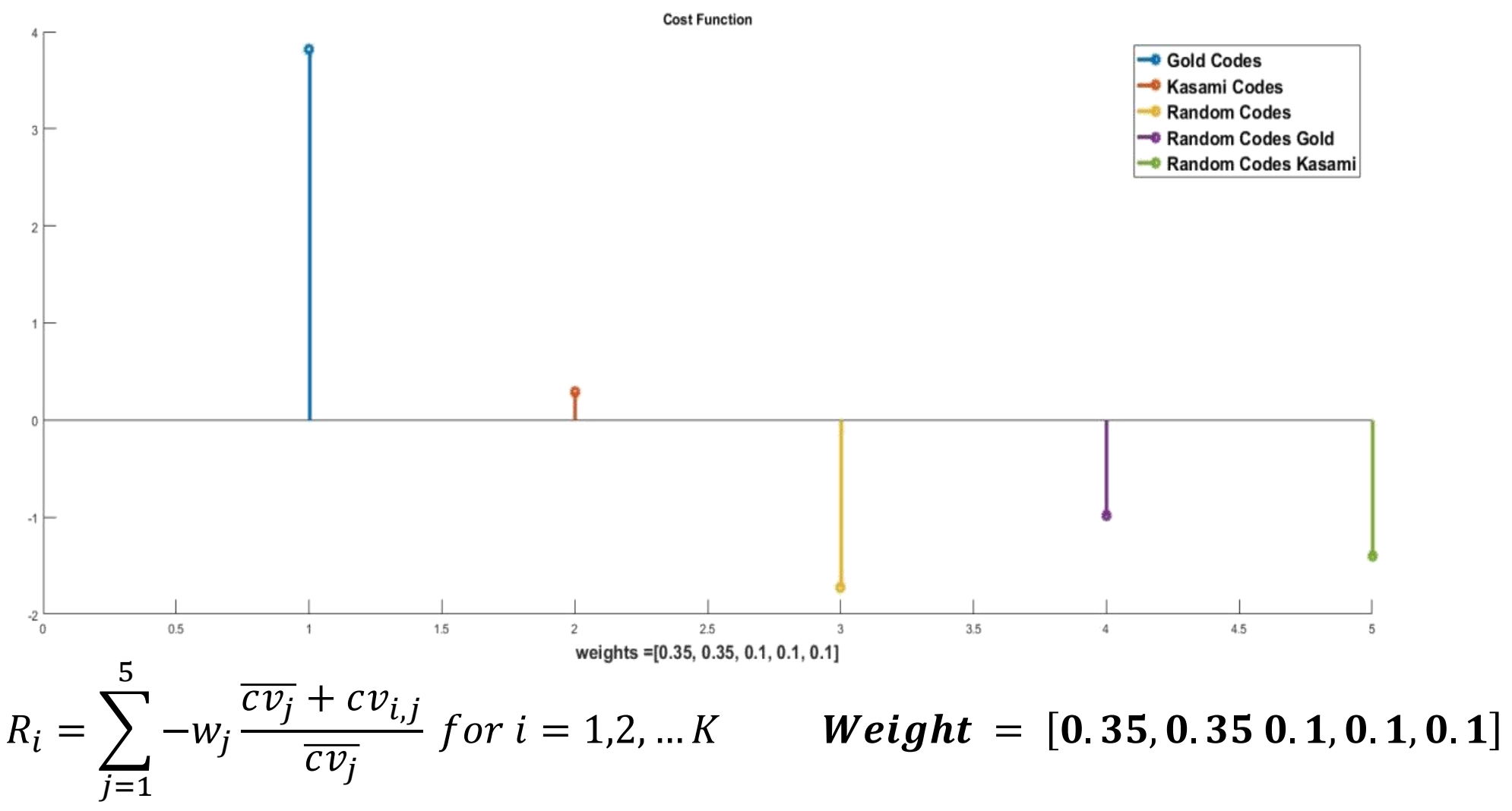


- Introduction \& background

- Design a new modulation for fast acquisition:

- BCS

- Design new PRN codes for fast acquisition:

- Random codes

- Co-design of the message structure and channel coding:

- Maximum distance separable codes(MDS)

- Full diversity Codes

- Conclusion

- Future Lines 


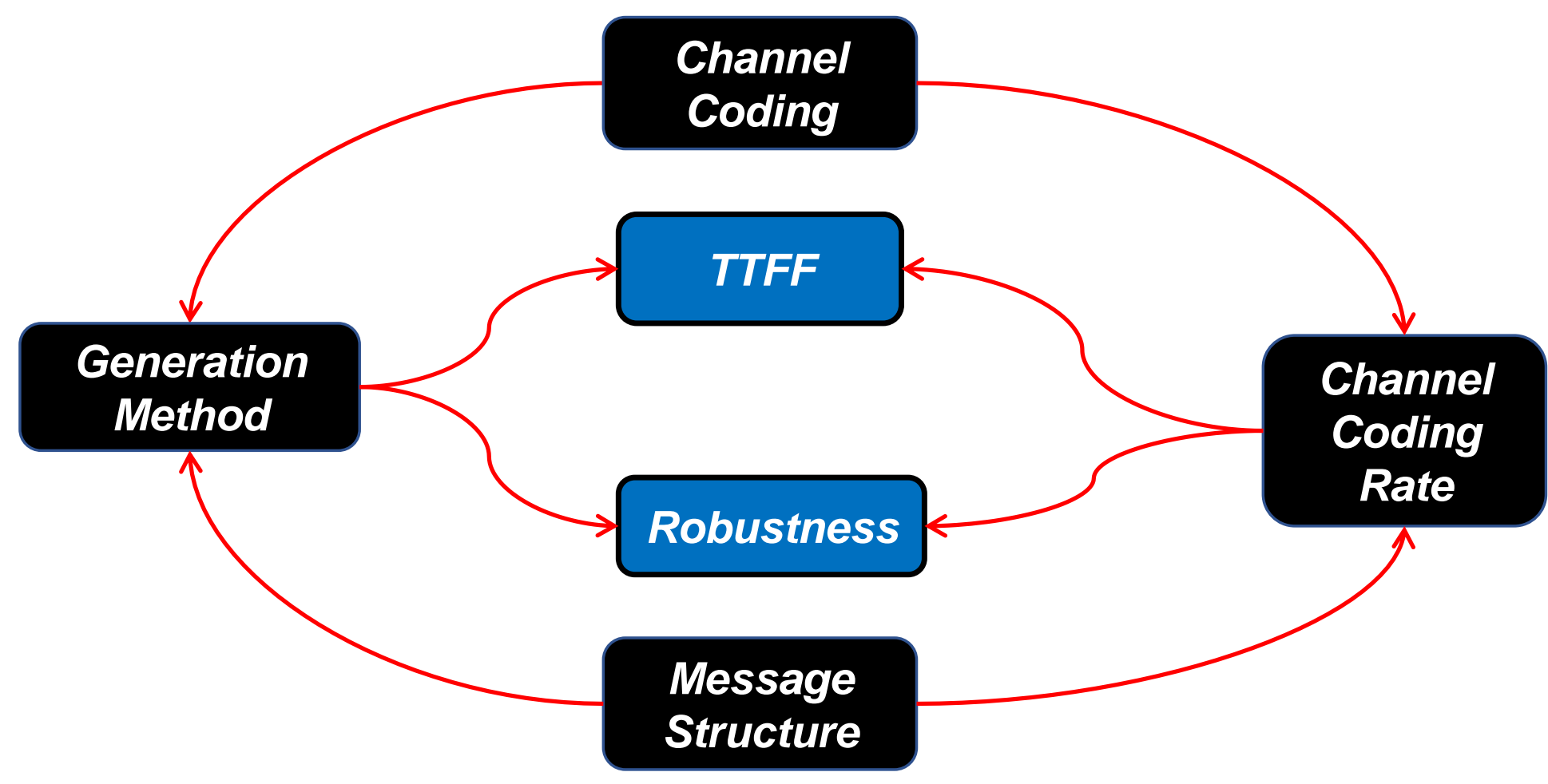


- What do we need?

- CRC (Cyclic Redundancy Check) which provides information about the integrity of the message.

- Good error correction capabilities under harsh channel conditions.

- Under good channel conditions, be capable of retrieving the CED with just the CED redundancy data (thanks to this capability we are able to reduce the $T_{C E D}$ ). 


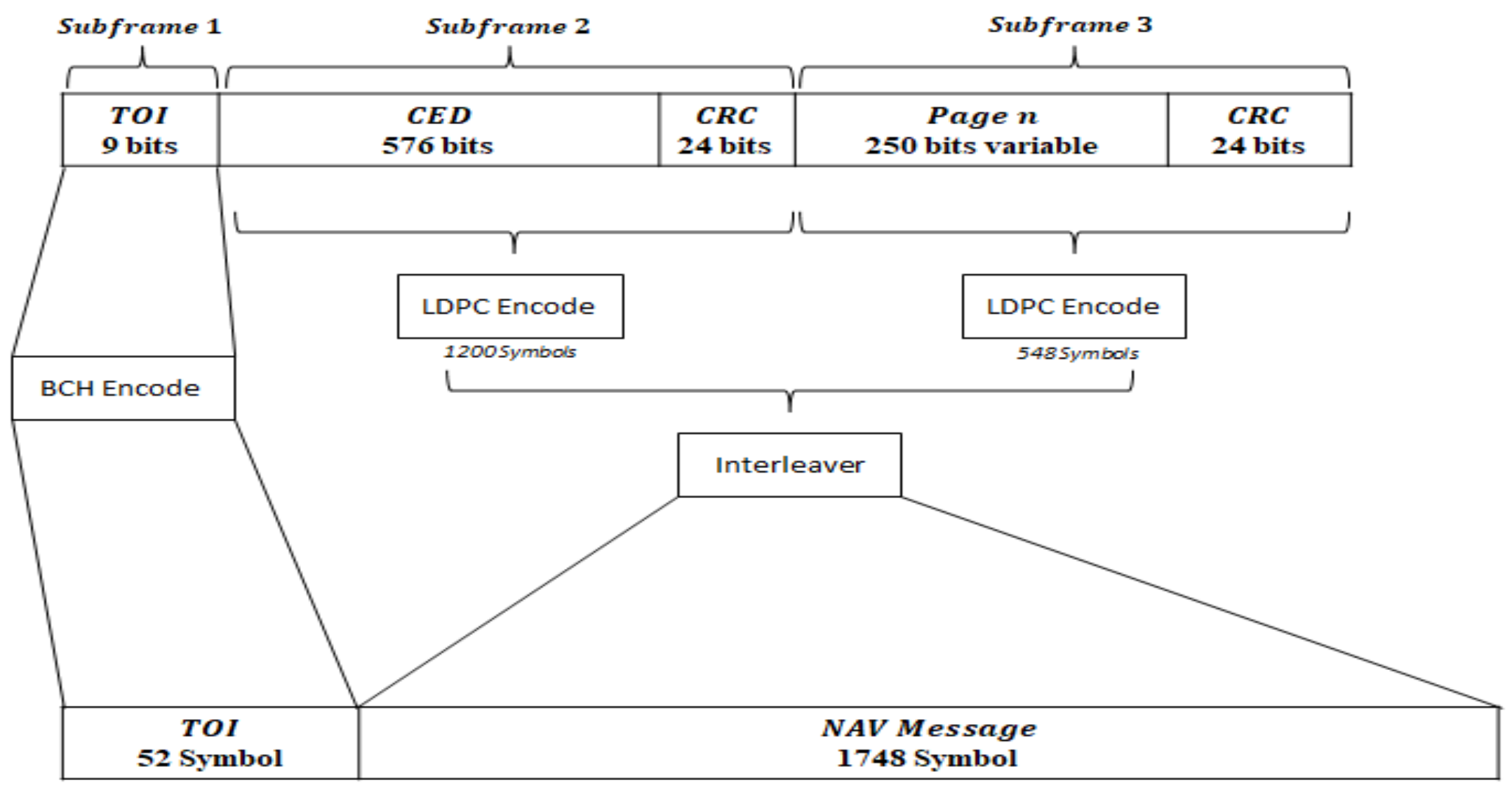

GPS L1C Message Structure 
Block fading channel model

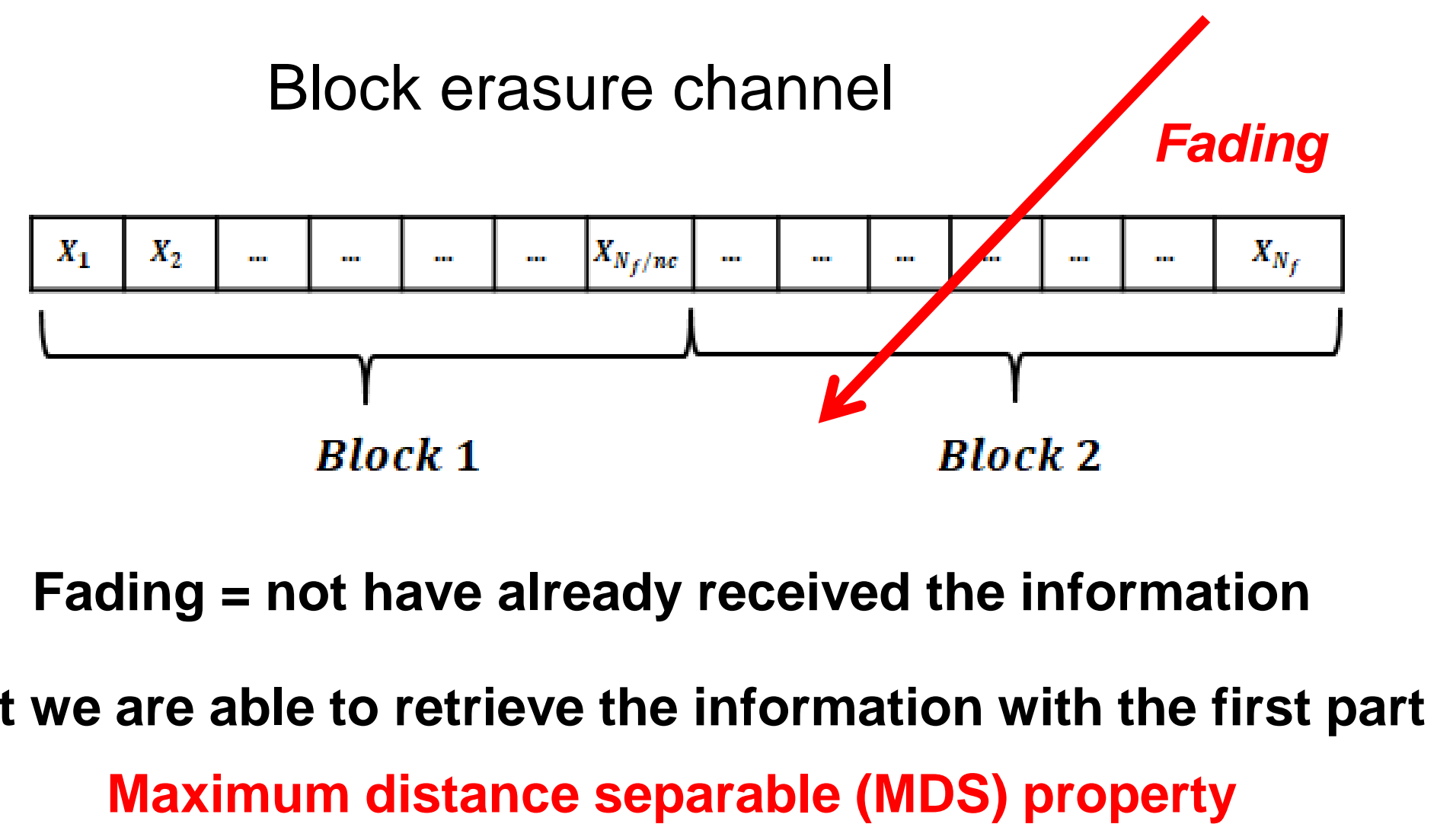


Block fading channel model
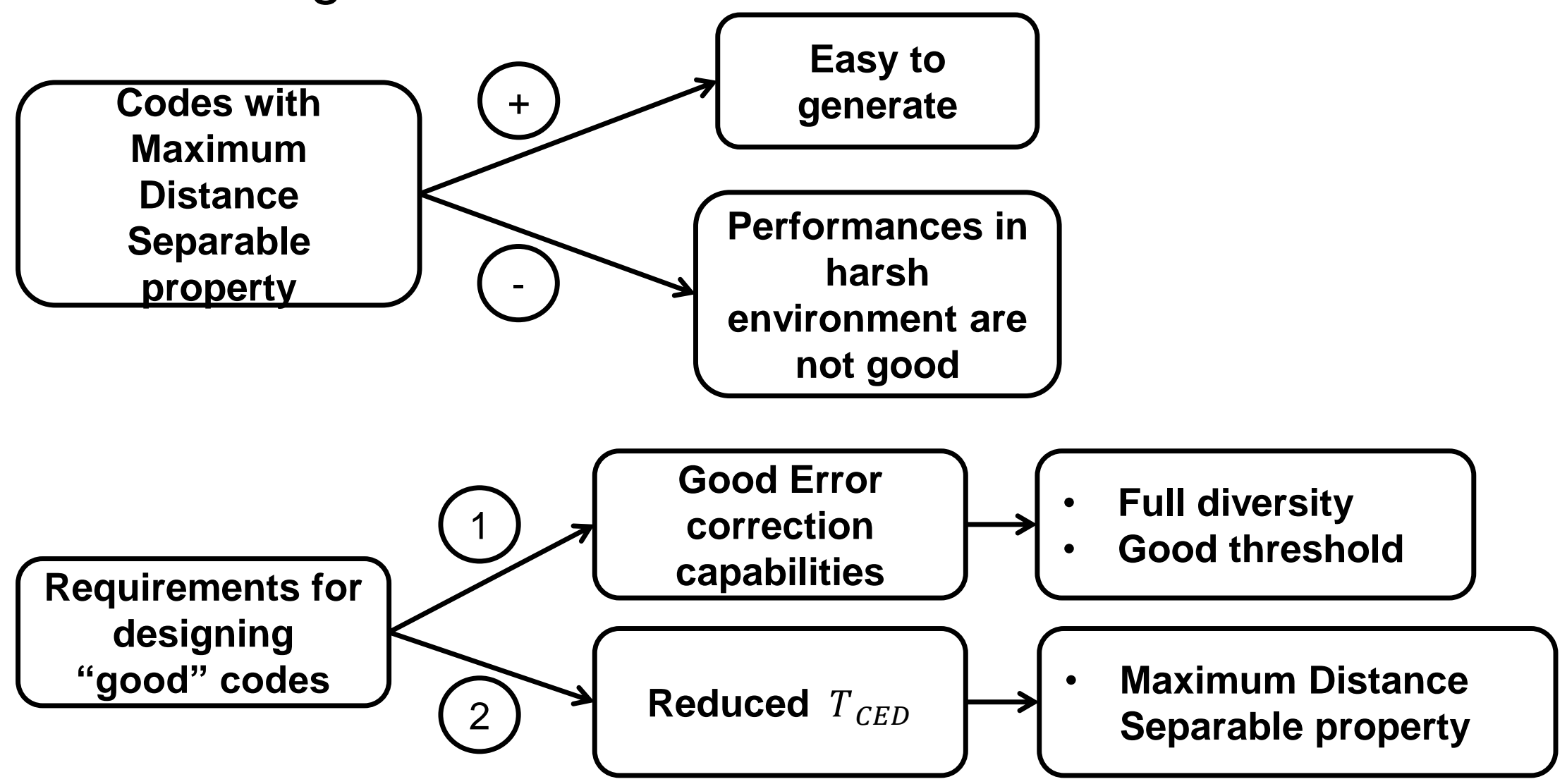
- Lowest Density-Maximum Distance Separable (LD-MDS) codes

- Maximum Distance Separable (MDS) codes

- Regular Root codes 
LD-MDS codes

Example:

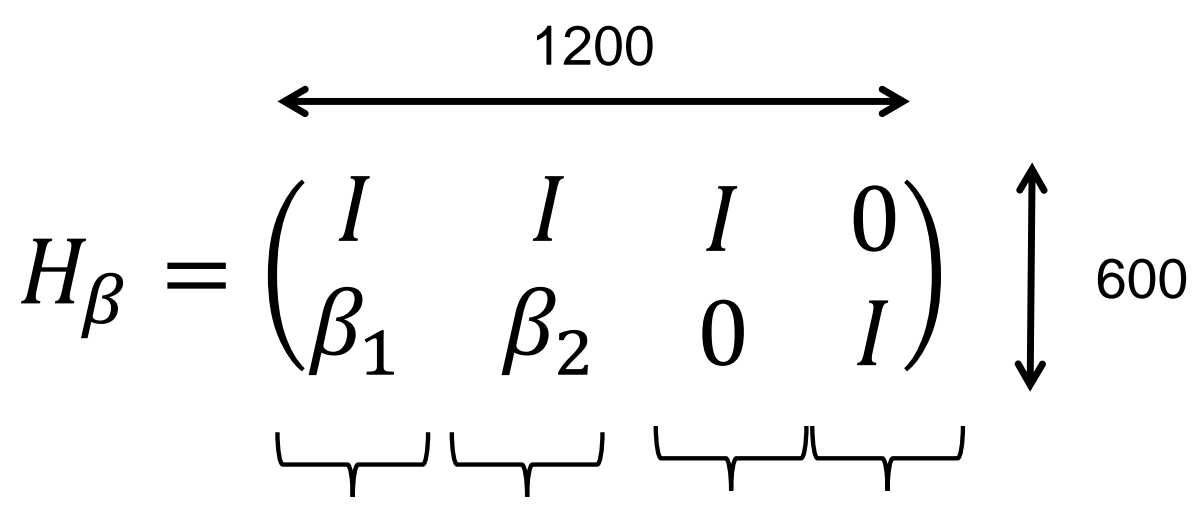

$$
\begin{aligned}
& \text { k1 } \quad k 2 \quad k 3 \quad k 4
\end{aligned}
$$

With any 2 error-free-blocks we retrieve the information $\rightarrow$ Erasure algorithm In case of errors with more than 2 blocks $\rightarrow$ BP Algorithm 


\section{LD-MDS codes}

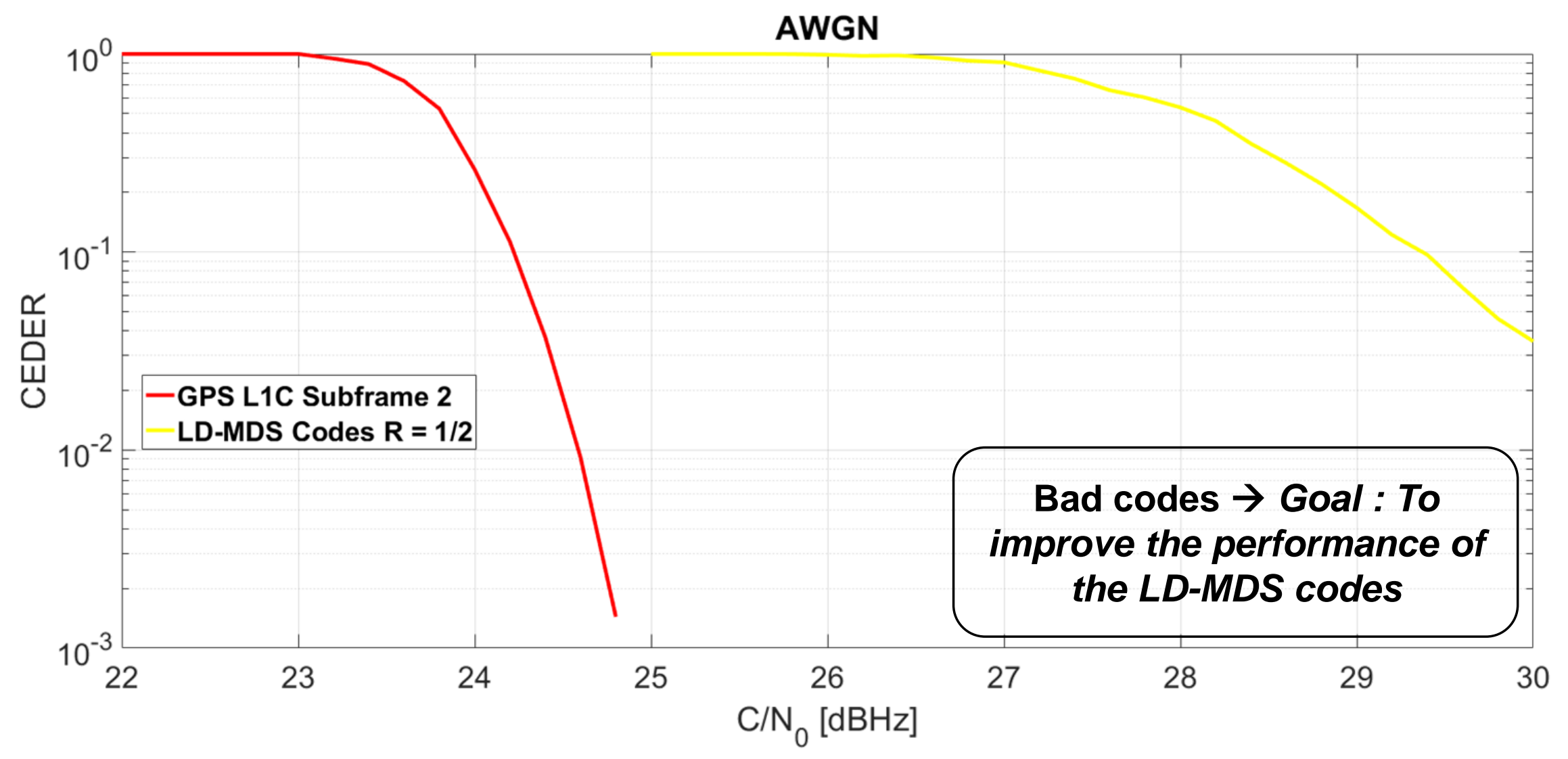


MDS codes

Example:

$$
H_{\beta}=\left(\begin{array}{llll}
\stackrel{4}{\beta_{1}^{\prime}} & \beta_{2}^{\prime} & I & 0 \\
\beta_{1} & \beta_{2} & 0 & I
\end{array}\right) \varlimsup_{600}
$$

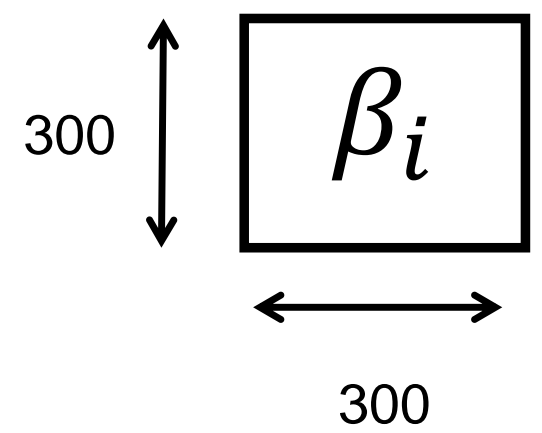


MDS codes

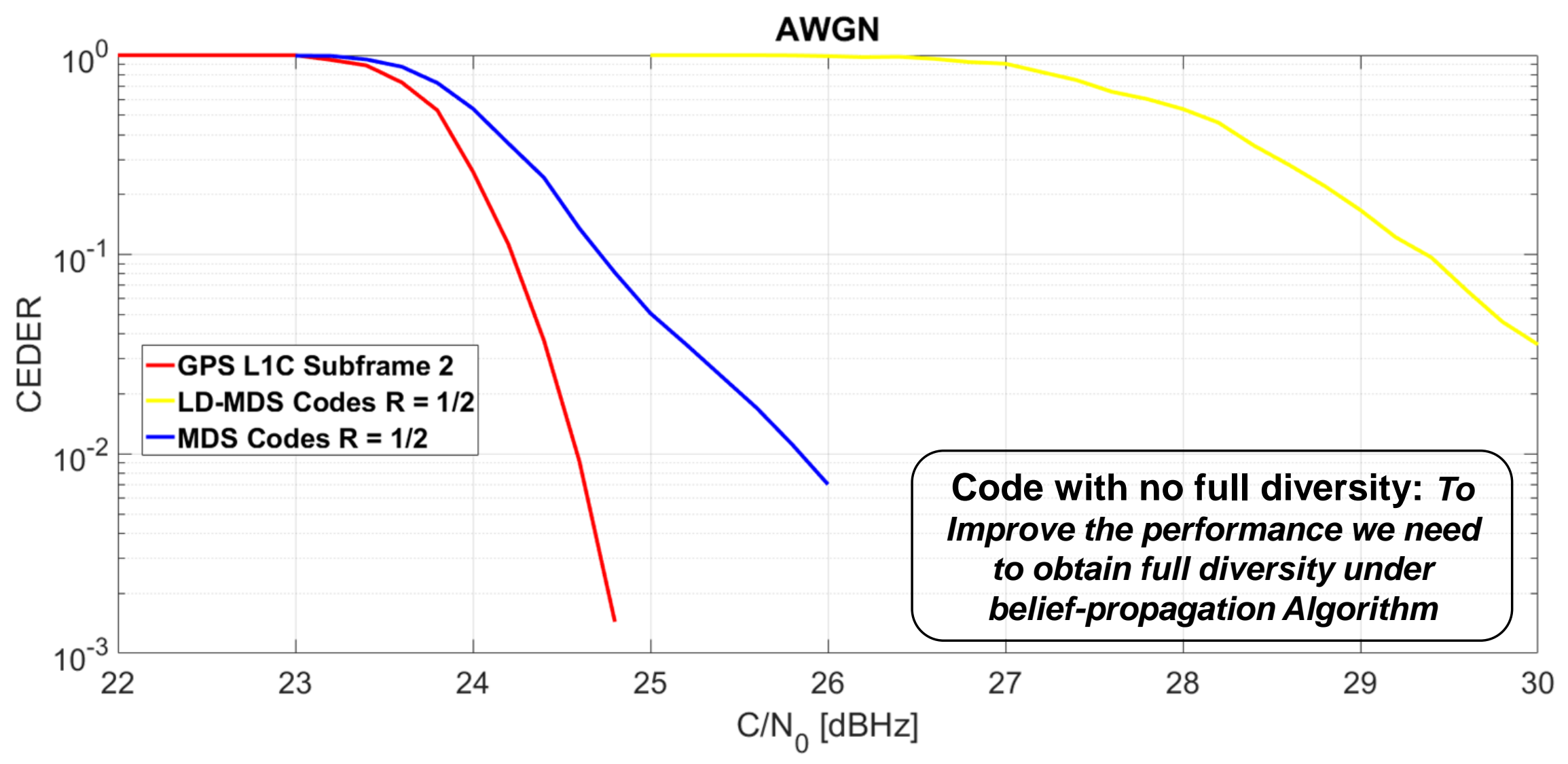


Regular-Root codes

They have full diversity property under BP algorithm

Example:

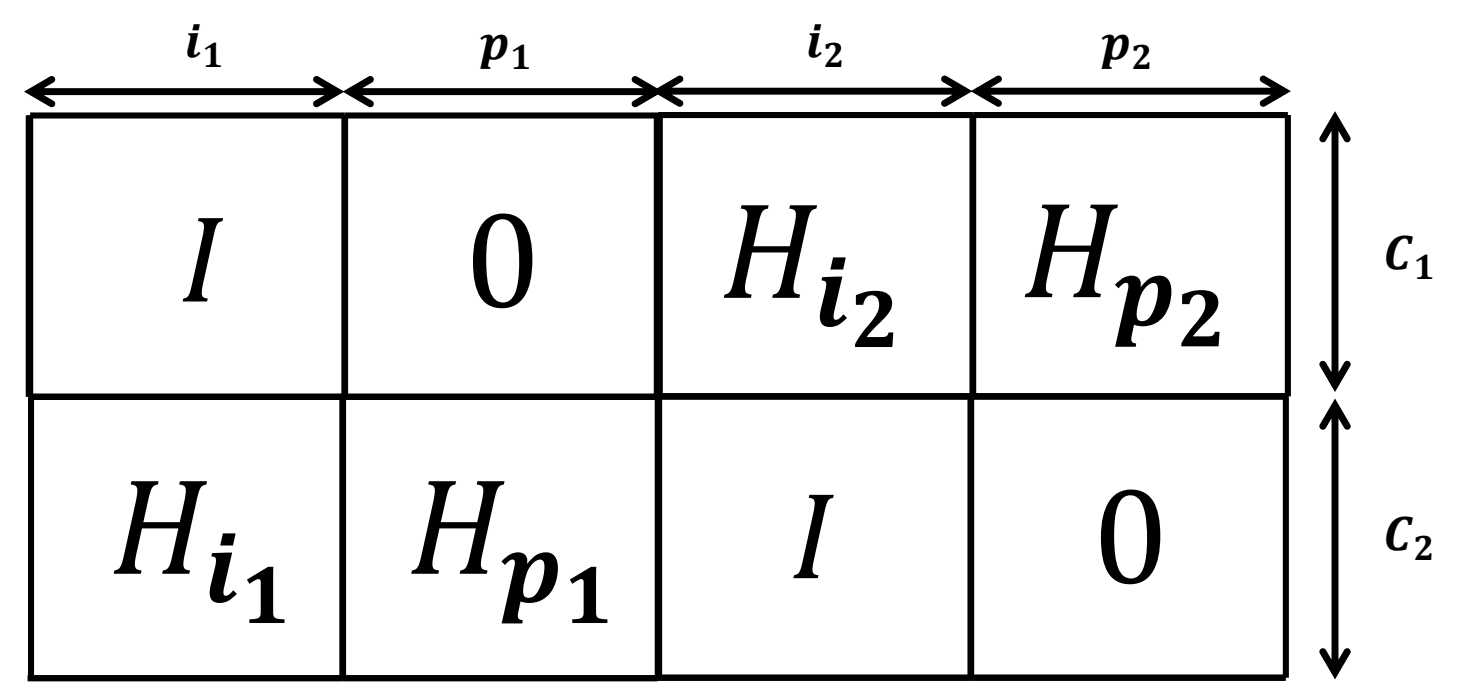

We don't need erasure algorithm $\rightarrow$ Running BP algorithm, we retrieve the information

Fading 1

Fading 2 


\section{Regular-Root codes}

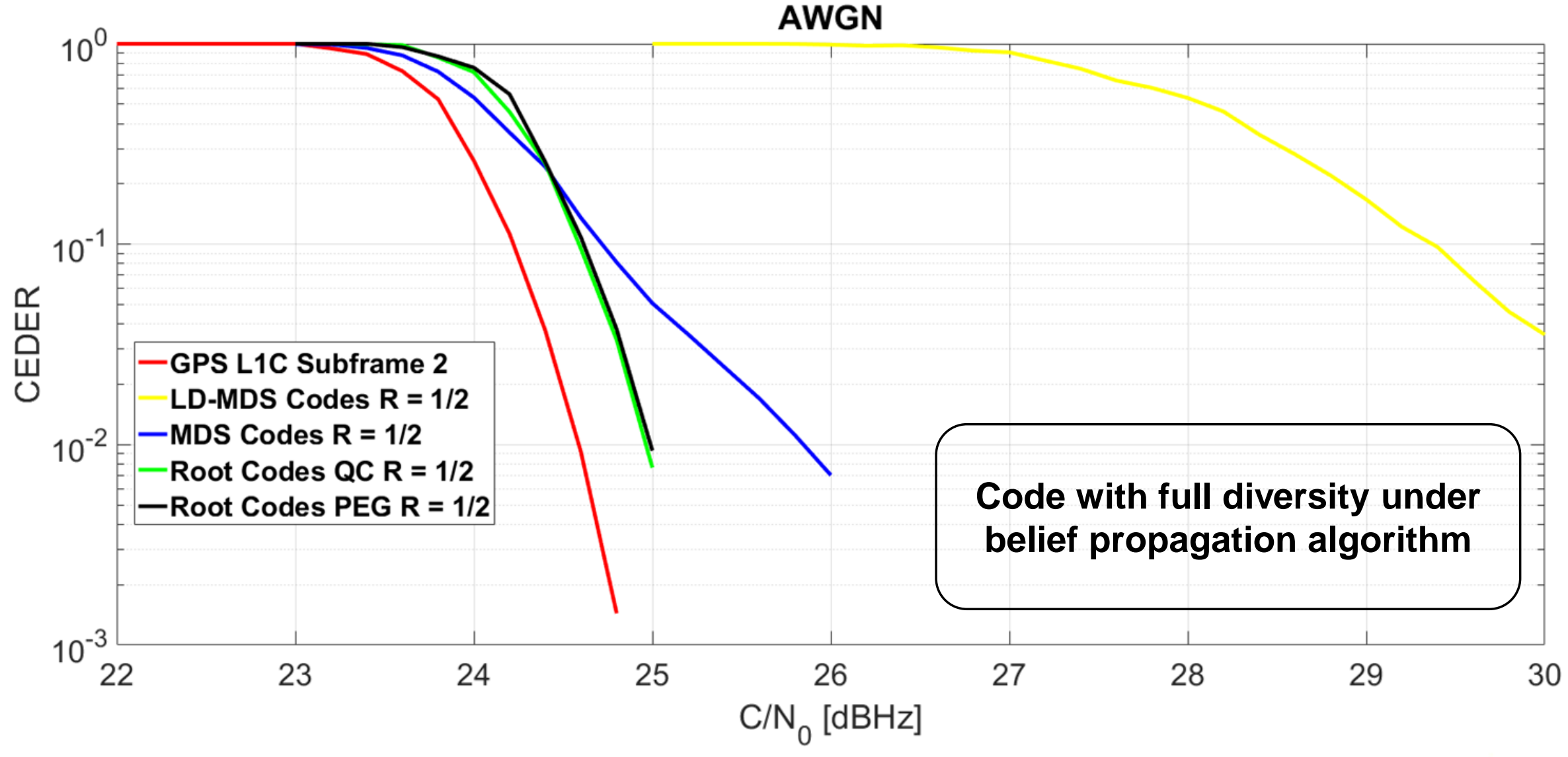




\section{Cumulative Distribution Function (CDF)}

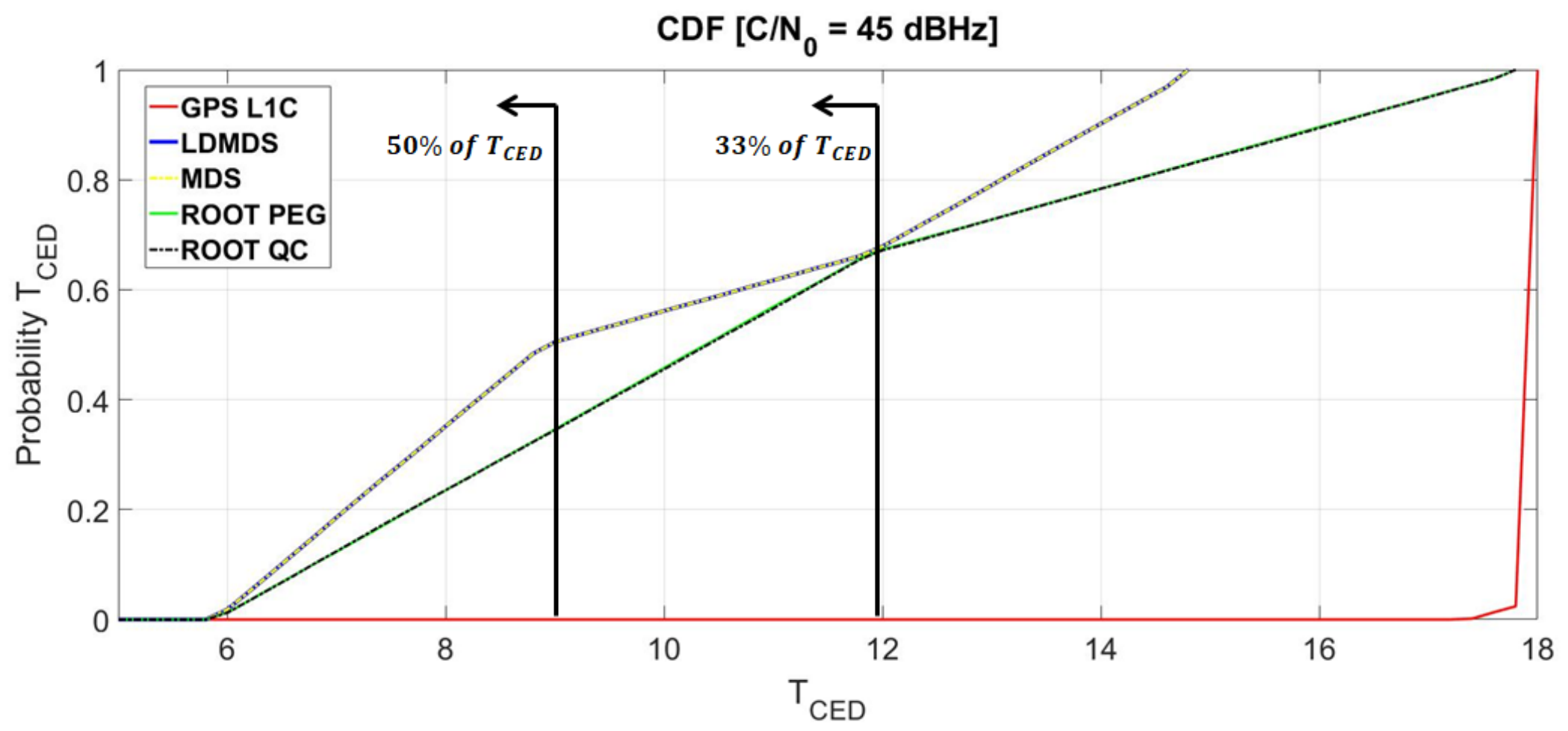

Under good channel conditions, we reduce the $T_{C E D}$ 


\section{Cumulative Distribution Function (CDF)}

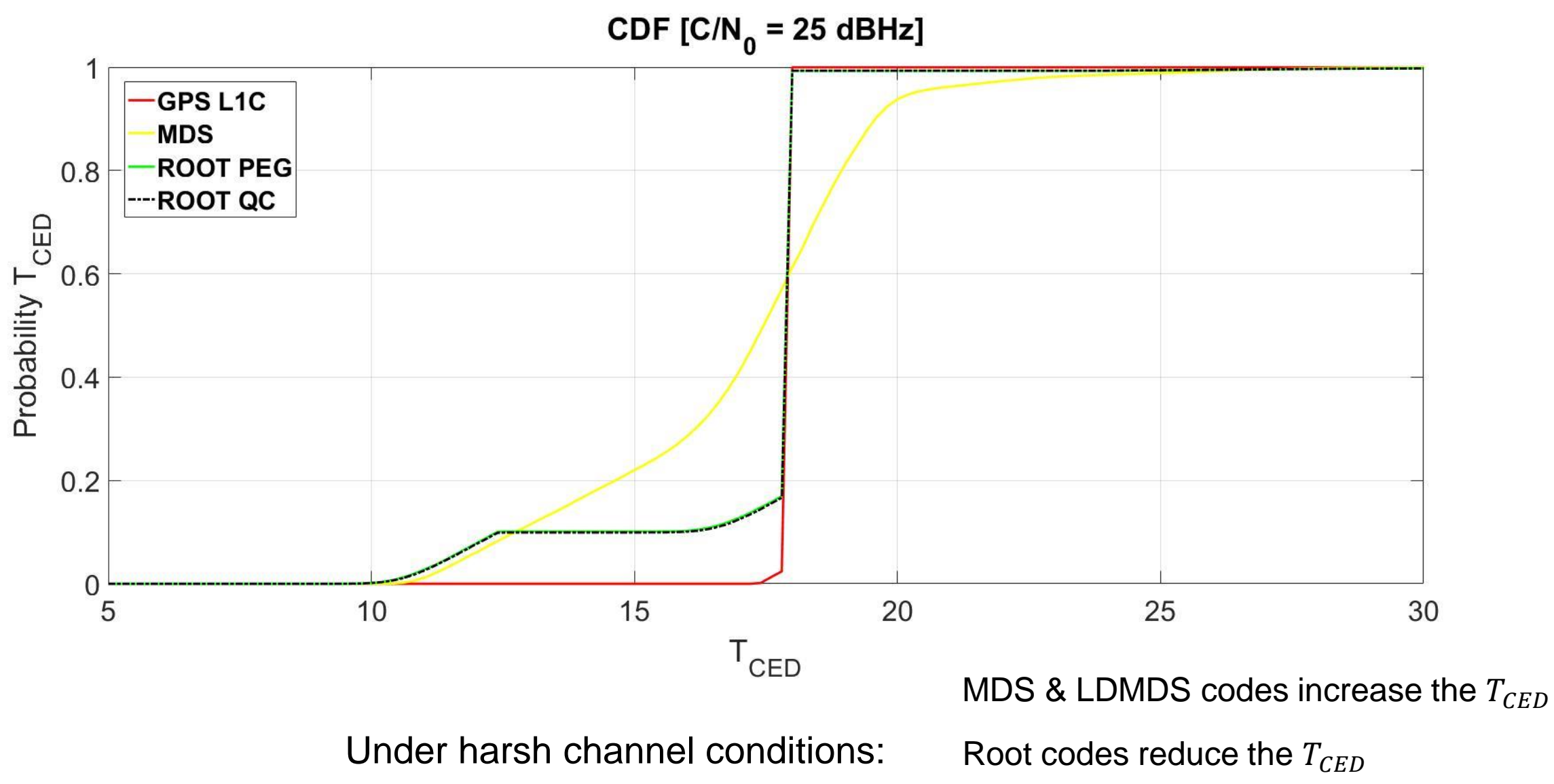




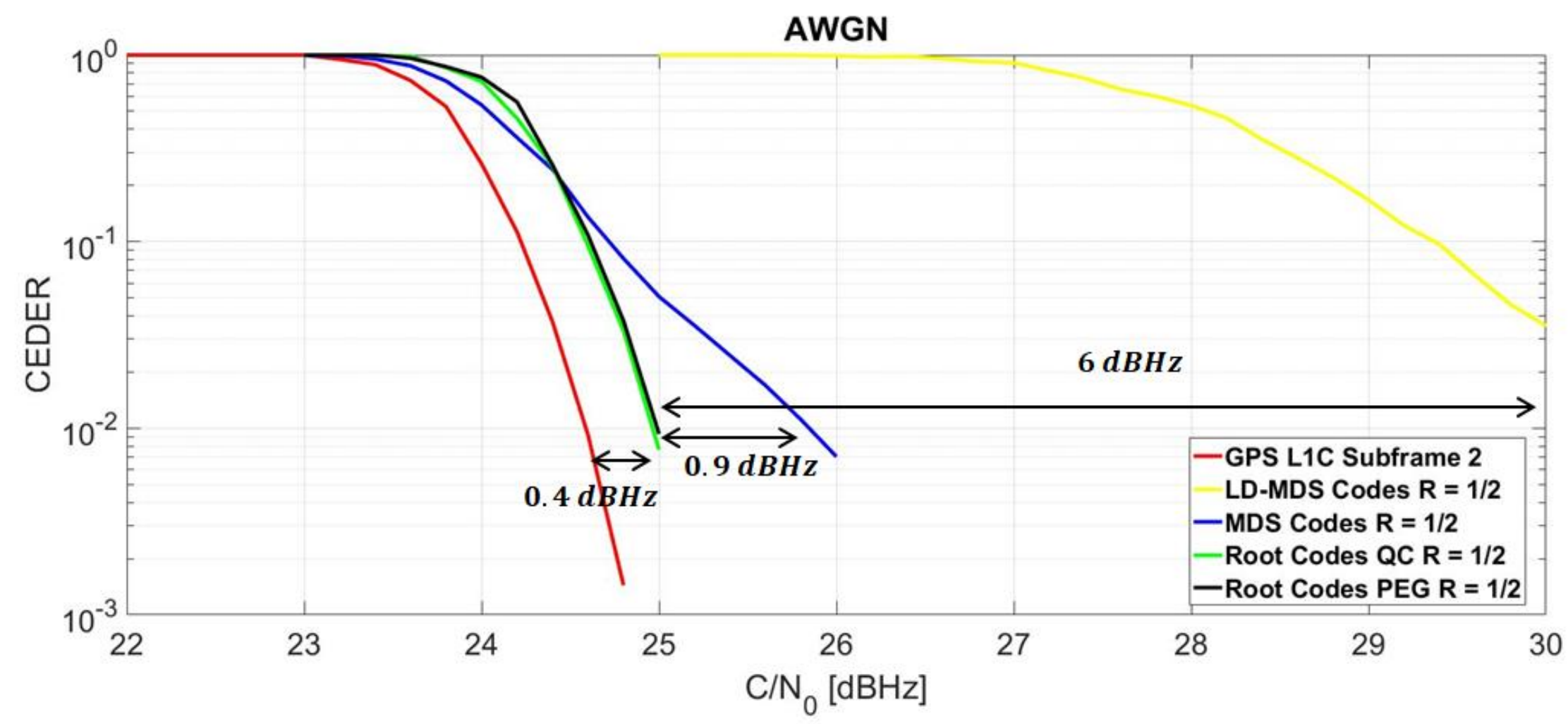


- Introduction \& background

- Design a new modulation for fast acquisition:

- BCS

- Design new PRN codes for fast acquisition:

- Random codes

- Co-design of the message structure and channel coding :

- Maximum distance separable codes(MDS)

- Full diversity Codes

- Conclusion

- Future Lines 
- Objective : Design a new signal to improve the acquisition phase

- To improve the receiver sensitivity and to reduce the TTFF*

- Results:

○ $\mathrm{BCS}[-1,-1,-1,1,-1](1) \longrightarrow$ Improve of the receiver sensitivity

- Random Sequences PRN codes

- Regular root-codes:

- MDS property under BP algorithm

- Full diversity

Reduction of the TTFF and easing the acquisition phase vs GPS L1C

High Reduction of the TTFF with good data demodulation vs GPS 
- Introduction \& background

- Design a new modulation for fast acquisition:

○ BCS

- Design new PRN codes for fast acquisition:

- Random codes

- Co-design of the message structure and channel coding :

- Maximum distance separable codes(MDS)

- Full diversity Codes

- Conclusion

- Future Lines 
- Next step:

○ Multiplexing the new signal 
thank you!

un del $ه$ 